\title{
Highly efficient and reusable energy-absorbing metamaterials exploiting soft rate-dependent frictional interfaces
}

\section{Jinyou Li}

Tsinghua University

\section{Zhe Chen}

Tsinghua University

Qunyang Li

Tsinghua University https://orcid.org/0000-0002-6865-3863

Lihua Jin

University of California Los Angeles https://orcid.org/0000-0001-7812-9056

Zhihua Zhao ( $\nabla$ zhaozh@tsinghua.edu.cn )

Tsinghua University

\section{Article}

Keywords: energy-absorbing efficiency, reusability, soft elastomer

Posted Date: October 22nd, 2021

DOI: https://doi.org/10.21203/rs.3.rs-968295/v1

License: (c) (1) This work is licensed under a Creative Commons Attribution 4.0 International License. Read Full License 


\title{
Highly efficient and reusable energy-absorbing metamaterials exploiting soft rate-dependent frictional interfaces
}

\author{
Jinyou $\operatorname{Li}^{1}$, Zhe Chen ${ }^{1}$, Qunyang $\mathrm{Li}^{* 1}$, Lihua Jin ${ }^{\dagger 2}$, and Zhihua Zhao ${ }^{\ddagger 1}$ \\ ${ }^{1}$ School of Aerospace Engineering, Tsinghua University, Beijing 100084, PRC \\ ${ }^{2}$ Department of Mechanical and Aerospace Engineering, University of California, Los Angeles, CA \\ 90095, USA
}

plateau. Achieving such curves is crucial for creating exceptional energy-absorbing materials, and has attracted great research interest in exploring original design strategies ${ }^{6,7}$.

The most well-known energy-absorbing mechanism is damaging constituent materials, such as ductile metals ${ }^{8,9}$ brittle foams ${ }^{10-14}$ and ceramics ${ }^{15,16}$. Besides, in order to maintain a long yielding force plateau, curved shapes ${ }^{17}$ or auxetic materials ${ }^{18}$ are introduced to prevent structures from immediately losing their load-carrying capabilities due to localization. The mechanisms of damage and plastic flow can dissipate a huge amount of energy, benefited from bond breakage or dislocations motion at the molecule level. Taking commercial aluminum foams as an example $^{19,20}$, their energy-absorbing capacities are as high as $30 \mathrm{~kJ} / \mathrm{kg}$ or $30 \mathrm{MJ} / \mathrm{m}^{3}$. The excellent performance has endowed them with broad applications in engineering, such as protecting cargo $^{21}$ and preventing collapse of rocks in mining $^{22}$. However, they are usually for one-time usage, after which the constituents are permanently damaged. This shortcoming can be partially overcome by incorporating damage-tolerant micro-lattices ${ }^{23-27}$ or phase-transforming constituents $^{28-30}$ that allow the materials to undergo cyclic loadings, although the performance decreases along cycles.

To completely remove the one-time-usage limitation, a promising way is designing the microstructures of metamaterials, which provide a vast space to gain outstanding mechanical properties that are otherwise hard to achieve ${ }^{31-34}$. By introducing non-damage energy dissipation mechanisms into microstructures, researchers have developed reusable energy-absorbing metamaterials. A well-investigated mechanism is mechanical instability of micro-cells, such as buckling of flexible beams ${ }^{35-39}$ and shells ${ }^{40}$, and nonlinear forces between magnets ${ }^{41-43}$. The assembled structures, obtained by connecting a series of these micro-cells, often produce hysteric saw-tooth force-displacement curves $^{44,45}$. The metamaterials constructed in this way are reusable since the deformation is elastic. Nevertheless, their energy-absorbing capabilities are typically several orders of magnitude lower than those of the non-reusable ones, which significantly 
1 limits their potential applications. For example, one kind of micro-beam based metamaterial ${ }^{46}$ only has an energyabsorption capacity around $0.15 \mathrm{~kJ} / \mathrm{kg}$ or $0.015 \mathrm{MJ} / \mathrm{m}^{3}$.

The relatively low performance of reusable energyabsorbing metamaterials is mainly caused by two reasons: the constituent materials can only sustain limited forces, and only a small portion of the microstructures contribute to energy dissipation. A strategy to improve the capacity is increasing the peak forces of the saw-teeth ${ }^{47-49}$ through increasing the maximum stresses and using stiffer materials. However, this method endangers both the constituent materials and protected targets. Researchers recently attempted other non-damage energy dissipation mechanisms, such as visco-elasticity ${ }^{50}$ and dry friction ${ }^{51-53}$ between sliding particles, to build energy-absorbing metamaterials. Nevertheless, their designs are still in infancy and the obtained energy-absorption capacities are as low as other reusable ones. Therefore, it is still an open problem to simultaneously achieve high-reusability and high-capacity metamaterials for energy absorption (Fig. 1a). a

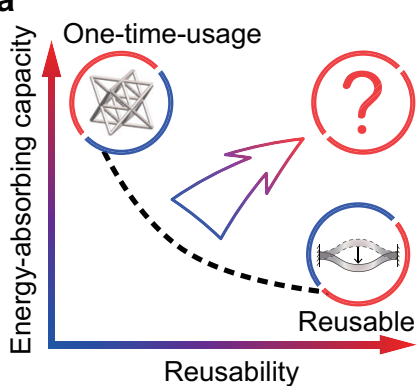

C

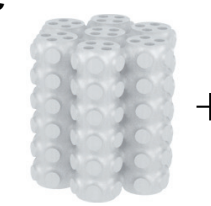

Soft elastomer

d
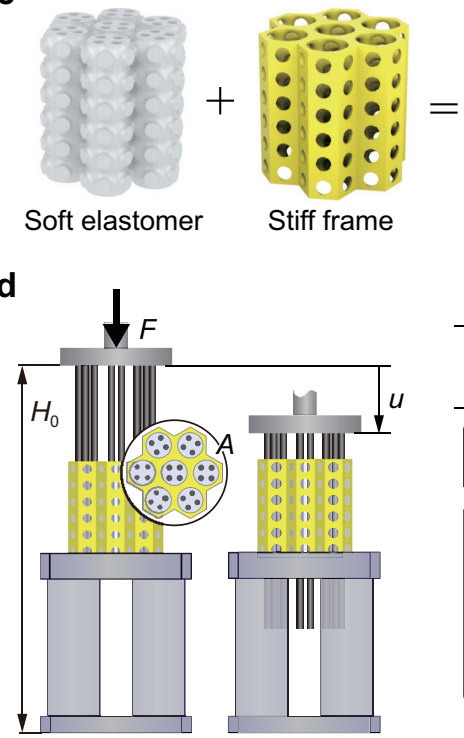

Stiff frame

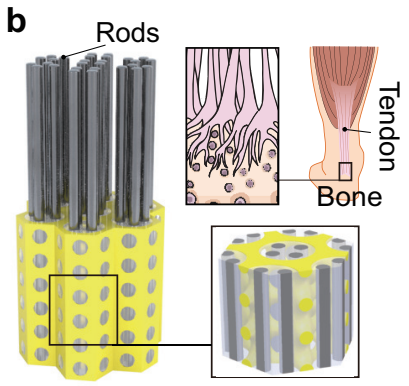

Interwoven structure
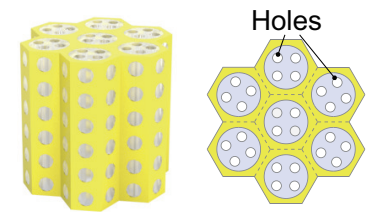

Reinforced elastomer
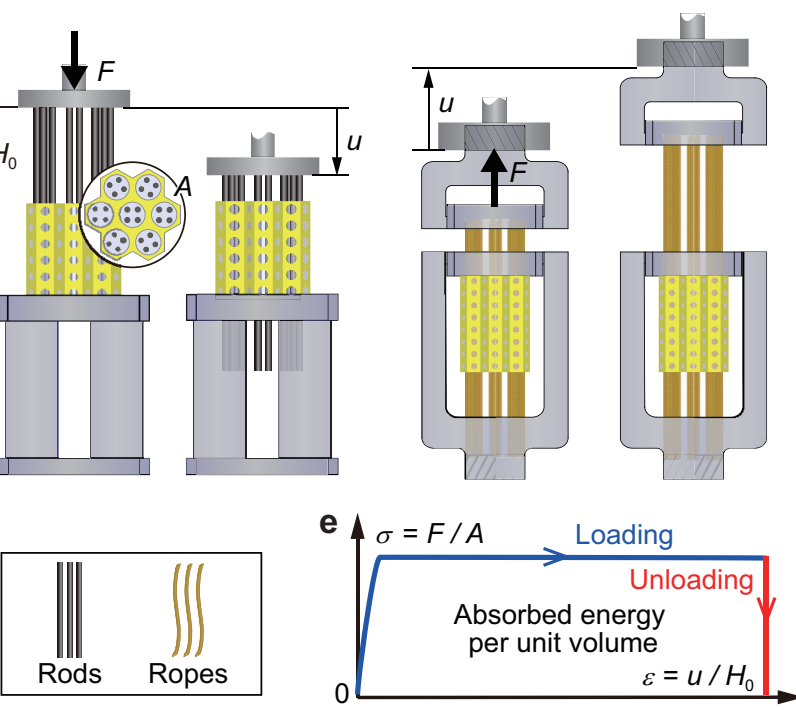

Fig. 1: Design of the proposed energy-absorbing metamaterials with both high capacity and high reusability. a A typical energy-absorbing material shows a trade-off between reusability and energy-absorbing capacity. b-c The proposed metamaterial is composed of stiff rods/ropes and a porous reinforced elastomer, which can slide between each other. The porous reinforced elastomer has a stiff frame interwoven with a soft silicone elastomer, inspired by tendon-bone interface shown in upper right corner of (b). d The metamaterial can be subjected to compression or tension. Here, $H_{0}$ is the total height, including the supporting platform, and $A$ is the crosssectional area of the reinforced elastomer. e The measured force balances the friction forces between the rods/ropes and reinforced elastomer. The force $F$ is expected to first sharply increase with the displacement $u$ in the static friction region, and then reaches a plateau in the dynamic friction region, which shows an ideal rectangular shape for energy absorption.

\section{Results}

Interwoven structure of the proposed metamaterials. Figure 1b sketches the overall design of the proposed metamaterials. We interwove a soft porous silicone elas- 
a
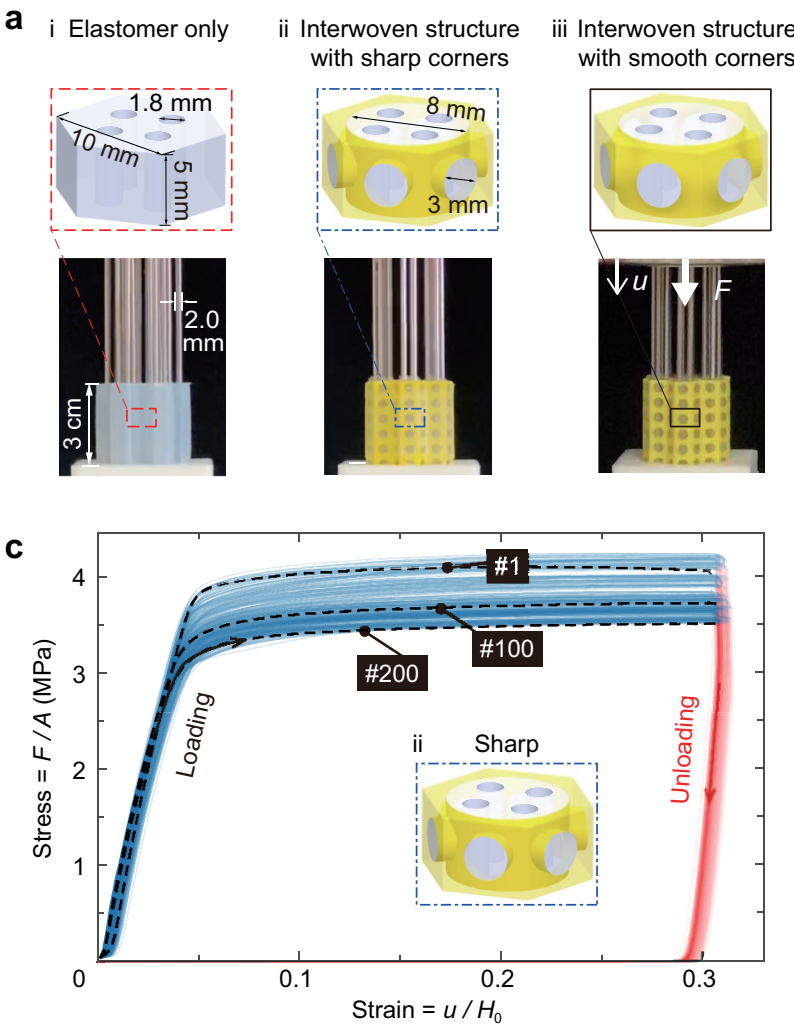
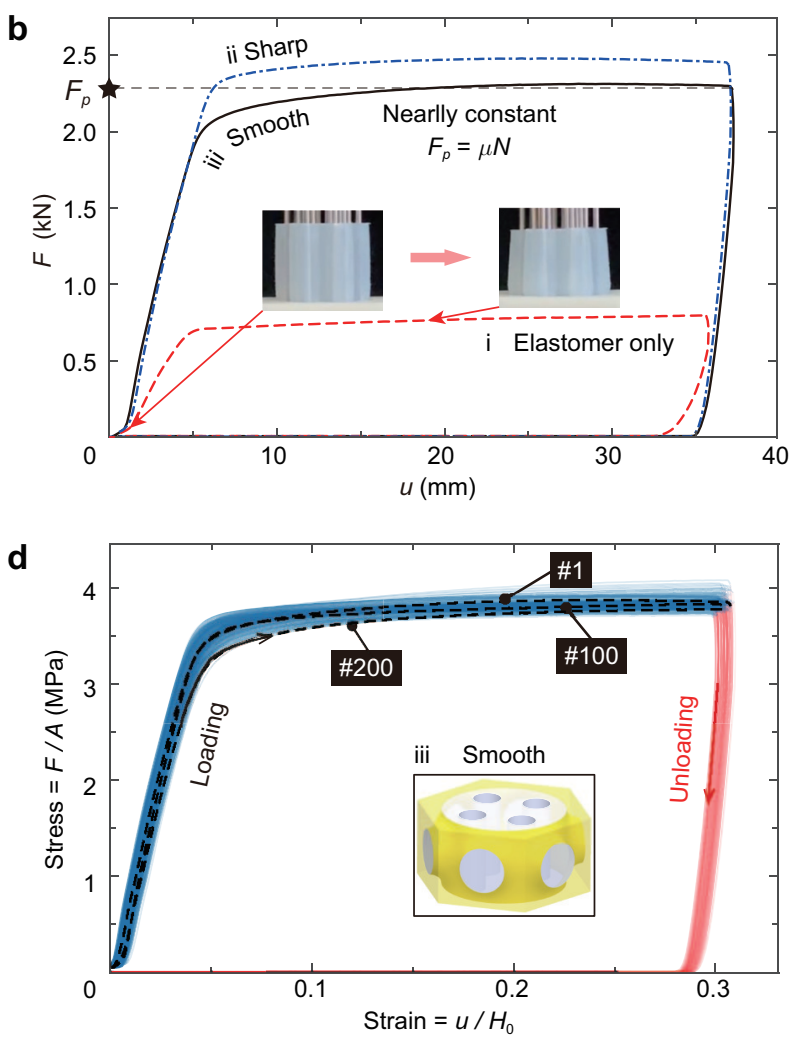

Fig. 2: Experimental verification of high capacity and reusability of the proposed energy-absorbing metamaterials. a Three different structures, stacked up by cell i, ii and iii, to understand how the prestress of elastomer and stiffness of the supporting frame affect the energy-absorbing performance. The upper schematics display the structure of each cell. Cell i contains only an elastomer, ii and iii involve stiff frames with sharp and smooth corners, respectively, to reinforce the elastomer. Each cell has a height $5 \mathrm{~mm}$ and flat-to-flat distance $10 \mathrm{~mm}$. The diameter of the rods $2.0 \mathrm{~mm}$ is slightly larger than that of the holes $1.8 \mathrm{~mm}$ to create pressure between the elastomer and the rods. The lower images show the corresponding manufactured seven-column samples. b Measured load-displacement curves for structures in (a) with a compression loading rate $v=500 \mathrm{~mm} / \mathrm{min}$. All the curves show nearly flat force plateaus $F_{p}$ when the displacement is high. The two metamaterials with interwoven structures exhibit higher $F_{p}, 3$ times as many as that of the one with only an elastomer. Inset: images showing the elastomer without a supporting frame is more compliant and undergoes a larger deformation. c-d Stress-strain curves of structure ii and iii under 200 loading-unloading cycles in reusability test. After all cycles, the capacity of the former decreases by about $14 \%$, while that of the latter varies only about $4 \%$.

tomer structure (gray) with a stiff porous polylactic acid (PLA) frame (yellow) to obtain a reinforced elastomer that has a better load-bearing capability. This interwoven structure was inspired by human's tendon-bone interface ${ }^{54,55}$, where soft tendon tissue interconnects with hard bone tissue to achieve a robust anchor (upper right part of Fig. 1b). The porous stiff frame was designed to have a number of hollow columns in a hexagonal packing, since a hexagonal packing has a better compression strength than a square or triangular one ${ }^{56}$ due to higher connectivity. Meanwhile, its surface was modified from a minimal surface to guarantee smoothness everywhere ${ }^{57}$, and avoid unnecessary stress concentrations. The PLA frame was 3D printed, while the porous silicone elastomer was cast using a mold composed of several parts (see Methods and Supplementary Fig. 1). More details on the structural geometry is given in Supplementary Fig. 2.

Steel or carbon fiber rods were inserted to the cylindrical holes in the reinforced elastomer. The diameter of the rods is slightly larger than that of the holes, which creates pressure between the elastomer and the rods along the frictional surfaces. When the rods are pushed downward (Fig. 1d), a compression force $F$ balances the friction forces. When $F$ is low, there is no relative displacement between the rods and the elastomer due to static friction, and $F$ linearly increases with the deformation of the elastomer. When $F$ is high, relative sliding between the rods and the elastomer occurs, and $F$ remains at a plateau corresponding to the dynamic friction force (Supplementary Movie 1). For the convenience of quantifying the energy-absorbing capacity, strain of the proposed metamaterials was defined as displacement $u$ divided by the initial height $H_{0}$ (Fig. 1d), and stress as force $F$ divided by the cross-sectional area $A$. Then, the stress-strain curve is expected as Fig. 1e, and the area of the stress-strain loop is the absorbed energy per unit volume. In addition, replacing the rods with 
ropes (right part of Fig. 1d) should yield similar frictional behavior against tensile loads.

Prestress of the elastomer and high stiffness of the supporting frame contribute together to high energy-absorbing capacity and reusability. As shown in Fig. 2a, we fabricated three different structures to reveal how the soft elastomer and stiff frame contribute to the energy-absorbing performance. All the structures have seven columns, arranged as Fig. 1c, and each column is stacked up by six regular hexagonal cells of height $5 \mathrm{~mm}$ and flat-to-flat distance $10 \mathrm{~mm}$. All the cells have four straight holes of diameter $1.8 \mathrm{~mm}$. The differences among the cells of the three structures are: cell $i$ is composed of the elastomer only; cell ii adopts a stiff porous frame, in which cylindrical bridges with sharp corners are used to connect adjacent cells, to reinforce the elastomer; and cell iii has a smooth stiff frame.

Inserting 2mm-diameter steel rods into the $1.8 \mathrm{~mm}$ diameter holes introduces prestress between the rods and the elastomer. Pushing rods downward, Fig. 2b shows the obtained load-displacement curves, measured under displacement control by a single-axis MTS Testing Machine with a loading rate $v=500 \mathrm{~mm} / \mathrm{min}$. Each of these curves possesses a linearly increasing region, followed by a long plateau with plateau force $F_{p}$, just as expected. As a result, both structures ii and iii show a higher slope of the force-displacement curve in the linear region and a higher plateau force than those of structure i due to the reinforcement of the stiff frames. Besides, the plateau force $F_{p}$ of structure ii is slightly larger than that of structure iii, since the supporting frame of cell ii is stiffer. More quantitative, the energy-absorbing capacities of structure ii and iii are around $1.0 \mathrm{MJ} / \mathrm{m}^{3}$, which is about three times as large as that of structure $i$ and comparable to non-reusable energy-absorbing materials, such as aluminum foams.

Further, we checked the reusability of structure ii and iii through cyclic loading. After 200 stress-strain cycles at a loading rate of $500 \mathrm{~mm} / \mathrm{min}$, the energy-absorbing capacity of structure ii decreases by about 14\% (Fig. 2c), which might be caused by the stress concentration in the elastomer and potential debonding between the soft elastomer and stiff frame due to sharper corners. However, energy-absorbing capacity of structure iii does not change notably (Fig. 2d). Overall speaking, structure iii has better comprehensive performance on energy-absorbing capacity and reusability, so that it was adopted here to design our metamaterials.

Load-displacement performance of a single column. Next, we would like to understand how physical parameters affect energy-absorbing capacity, which is mainly featured by $F_{p}$ of a single column. Obviously, $F_{p}$ can be modified by tailoring the parameters, e.g., the height $h$ of the elastomer, or the diameter $d$ of the steel rods (Fig. 3a). Theoretically, frictional dynamics says

$$
F_{p}=\mu N,
$$

where $\mu$ is the coefficient of dynamic friction between the elastomer and the rods, and $N$ is the total normal contact force acted on the rods. Intuitively, increasing the column height $h$ enlarges the contact area, and increasing the diameter difference between rods and holes $d-d_{h}$ enlarges the prestress. As a result, $N$, and therefore $F_{p}$, should be proportional to both $h$ and $d-d_{h}$, which was validated by varying a single geometric parameter in controlled experiments (Fig. 3b-c). To simplify the experiments, only four steel rods were inserted into the central column for each seven-column module (right in Fig. 3a); thus the obtained plateau values $F_{p}$ are about $1 / 7$ of that in Fig. 2b. Moreover, we observed $F_{p}$ increases notably with the loading rate $v$ (Fig. 3d), which indicates that energy-absorbing capability gets higher for a higher impact velocity. This attractive feature will be quantified and understood in the next few paragraphs.

Mechanisms of rate-dependent energy-absorbing behavior. The plateau force $F_{p}=\mu N$ relies on the loading rate $v$, indicating that at least one of $\mu$ and $N$ changes with $v$. Further quantitative studies reveal they both are, and only in that case can the velocity curve in Fig. 3d be understood. More specifically, we attribute the metamaterials' rate-dependent energy-absorbing behavior to the synergy of two iconic characteristics of elastomers: ratedependent frictional coefficient, and nonlinear hyperelastic property in large deformation.

Frictional coefficient $\mu$, especially for elastomers ${ }^{58}$, can be rate-dependent ${ }^{59-61}$; therefore, we first conducted friction experiments on a Tribometer (Rtec MFT-V) to quantify $\mu$ between our silicone elastomer and steel (see details in Supplementary Fig. 3). In the tribometer experiments, a steel sphere was pressed by a downward force $F_{z}$ onto an elastomer disc, placed on a rigid flat plate (inset of Fig. 3e). The plate was rotated to control the sliding speed, meanwhile, $F_{z}$ was maintained to be an almost constant. Considering that $\mu$ relies on normal contact stress ${ }^{62}$ and elastomer thickness, we prepared two elastomer discs of thicknesses $t=0.4$ and $1.3 \mathrm{~mm}$, since the thickness of the elastomer surrounding a single rod varies along the rod; and applied normal force $F_{z}=1.1$ and $3.0 \mathrm{~N}$ respectively, ensuring the nominal normal contact stress acted on the steel sphere approaches the average normal stress applied to the rods, which is about $0.5 \mathrm{MPa}$ according to the finite element method (FEM) simulations (for details see Supplementary Fig. 4), in which the constitutive behavior of silicone elastomer is assumed to follow the third order Ogden hyperelastic model ${ }^{63}$ (see Methods and Supplementary Fig. 5). Taking the ratio of the lateral force to the normal force at different loading rates $v$, the frictional coefficient $\mu$ versus $v$ can be obtained. Averaging the measured data of the two elastomer samples yields $\mu-v$ curve in Fig. 3e. When $v$ rises from 57 to $471 \mathrm{~mm} / \mathrm{min}, \mu$ increases by $30 \%$, while $F_{p}=\mu N$ increases by $60 \%$, greater than $\mu$.

Therefore, besides $\mu$, the normal contact force $N$ at the 

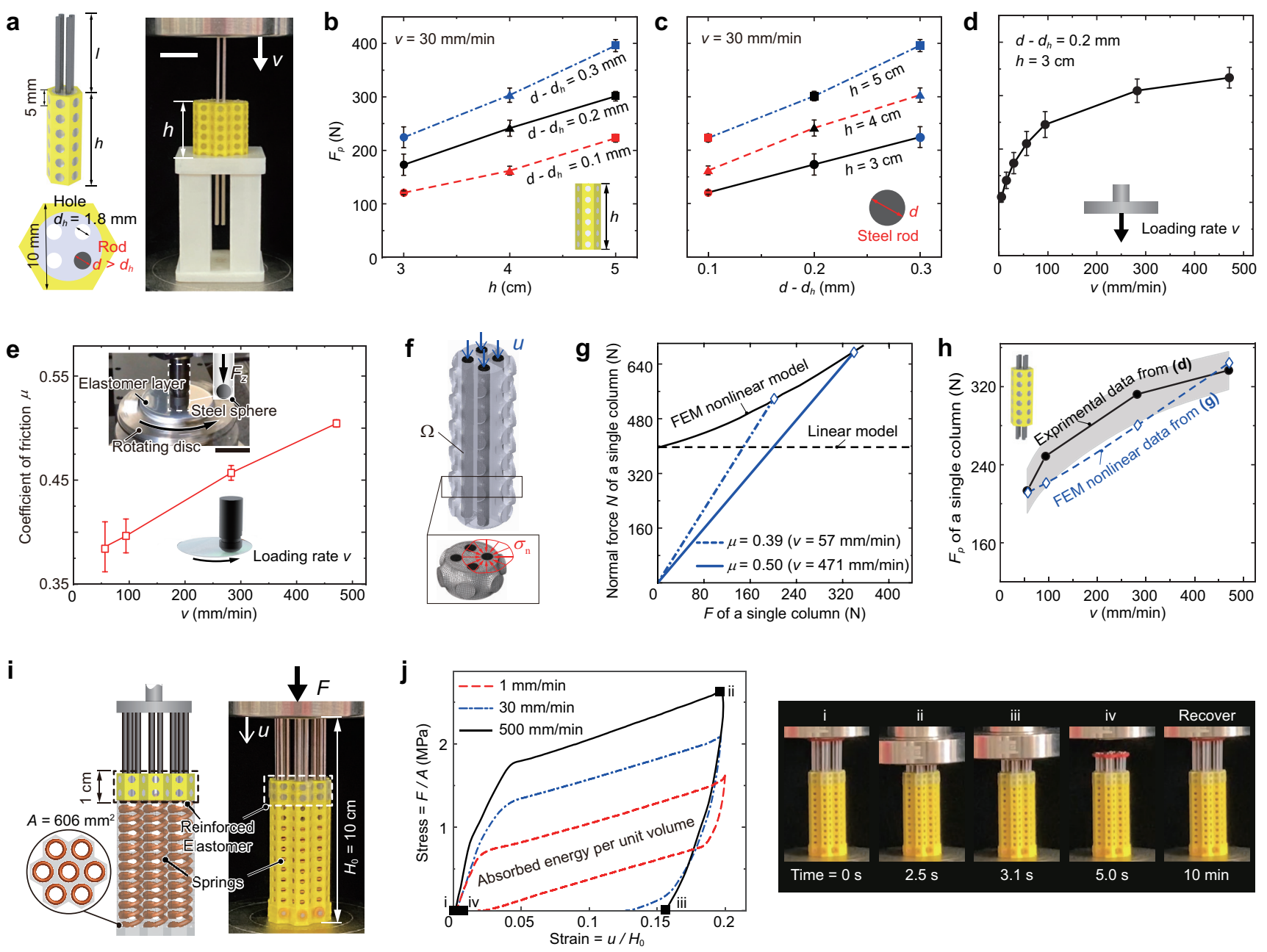

Fig. 3: Dependence of $F_{p}$ on geometric parameters and loading rate $v$. a Geometry of a single column of the reinforced elastomer (left), stacked up by multiple cells of type iii in Fig. 2a. The image (right) shows the experimental setup to quantify the performance of a single column under certain loading rate $v$, in which the scalar bar represents $2 \mathrm{~cm}$. b-d Dependence of $F_{p}$ on $h, d-d_{h}$ and $v$. The former two show linear relations, while the last one is nonlinear. For every data point, three samples were fabricated, and each sample was tested for four times; the error bars represent the ranges of the results obtained. e Measured $\mu-v$ curve between elastomer discs, with thickness 0.4 and $1.3 \mathrm{~mm}$, and a steel sphere by the experimental setup shown in the inset, where the scalar bar represents $2 \mathrm{~cm}$. The error bars represent the ranges of the results measured with different elastomer thickness. f FEM model of a single column, whose supporting frame is set to be invisible for clarity. The rods are forced to move downward by displacement $u$, the vertical reaction force $F$ is computed, and the normal force $N$ is obtained by integrating the normal stress $\sigma_{n}$ along the rods. $\mathbf{g} N-F$ curve calculated from the FEM model in (f). $\mathbf{h}$ Comparison of the experimentally measured $F_{p}-v$ curve (black solid) and the numerically calculated $F_{p}-v$ curve from the FEM model in (f-g). $\mathbf{i}$-j A self-recoverable energy-absorbing metamaterial, and its load-displacement curves under different loading rates. The right images show the configurations of the metamaterial corresponding to the different loading states indicated on the curve at a loading rate $v=500 \mathrm{~mm} / \mathrm{min}$.

steady sliding stage must also increase with $v$. We found this is implicitly caused by the combination of the geometric and material nonlinearity of the elastomer. When the soft elastomer around the holes is subjected to frictional shear forces $F$ from the rods, its large deformation pattern tends to shrink the holes, which in turn enlarges $N$. This was validated via a FEM model of a column of the metamaterial, as shown in Fig. 3f (for details see Methods and Supplementary Fig. 4). Rods and the elastomer were assumed to bond together. In quasi-static numerical simulations, each rod has a diameter of $1.8 \mathrm{~mm}$ in the stress-free state, and then was expanded to $2.0 \mathrm{~mm}$ to introduce prestress, after which the rods were forced to move downward by displacement $u$. Integrating normal stress over the interface $\Omega$ gives the normal force $N$. The obtained $N-F$ curve, black solid line in Fig. 3g, confirms that $N$ increases with $F$.

Combing the measured $\mu-v$ curve and the calculated $N-F$ curve, we can quantitatively explain the ratedependent behavior $F_{p}-v$ of the metamaterial. Under a given loading rate $v$, to reach the impending sliding condition, the normal and downward forces should sat- 
isfy $F-\mu(v) N=0$. On the other hand, $F$ and $N$ are intrinsically related due to the geometric and material nonlinearity, as predicted by the FEM model. As shown in Fig. $3 \mathrm{~g}$, under a given loading rate $v$, the linear relation $F=\mu(v) N$ can intersect with the nonlinear $F-N$ curve obtained by the FEM to determine the critical force $F_{p}(v)$ when impending sliding occurs. Several predicted values of $F_{p}$ at different $v$ are depicted by blue diamonds in Fig. 3h; they agree well with the measured force data.

Self-recoverable energy-absorbing metamaterials. The rate-dependent behavior of the friction force was utilized here to build a self-recoverable energy-absorbing metamaterial. As shown in Fig. 3i, we integrated each column of the previous metamaterial with an off-the-shelf coil spring, whose stiffness is $4.85 \mathrm{~N} / \mathrm{mm}$. Pushing the rods downward, the springs are compressed; and retreating the loading plate, the spring forces return the rods back against friction forces. It is expected that the return rate decreases as the spring forces decrease. Therefore, when the return rate of the rods is less than the unloading rate of the testing machine $v$, the loading plate detaches from the rods and the compression force $F$ drops to zero immediately (Fig. 3j and Supplementary Movie 2). After detaching, the rods can be further pushed back by the springs since the resistant friction forces decrease at lower rates (inset in Fig. 3j); this helps to extend the working stroke of the metamaterial.

The measured stress-strain curves at different rates in Fig. 3j confirm that a larger loading rate yields a larger hysteresis loop. At a loading rate of $500 \mathrm{~mm} / \mathrm{min}$, the energyabsorbing capacity is about $0.3 \mathrm{MJ} / \mathrm{m}^{3}$, which is about 20 times of that of the self-recoverable energy-absorbing metamaterials previously reported ${ }^{46}$.

Improving energy-absorbing capacity by lightweight constituent materials. Although the above metamaterials can absorb a large amount of energy, they are heavy since the rods are made of steel. Making use of the high frictional coefficients between silicone elastomers and other materials ${ }^{64}$, we replace the steel rods with other light-weight cores, such as carbon fiber rods and Kevlar ropes, to improve the energy-absorbing capacity per unit mass of the metamaterials. Consider a seven-column module (Fig. 4a) with the height of its elastomer $h$, mass density of the elastomer $\rho_{r}=6.6 \mathrm{~g} / \mathrm{cm}$, length of the cores $L$, and mass density of the cores $\rho_{c}=1.3$ and $0.56 \mathrm{~g} / \mathrm{cm}$ for carbon fiber rods and Kevlar ropes, respectively. If the magnitude of the friction force per unit length is $f$, the dissipated energy per unit mass $E_{\mathrm{m}}$ is:

$$
E_{\mathrm{m}}=\frac{f h(L-h)}{\rho_{r} h+\rho_{c} L}=\frac{f L}{\rho_{r}} \frac{\alpha(1-\alpha)}{\alpha+\rho_{c} / \rho_{r}}
$$

where $\alpha \triangleq h / L$ is the ratio of the elastomer height to the core length. For a given core, $E_{\mathrm{m}}$ monotonically increases with $L$. When $L$ is fixed, we define a dimensionless quantity $\bar{E}_{\mathrm{m}} \triangleq E_{\mathrm{m}} \rho_{r} /(f L)=\alpha(1-\alpha) /\left(\alpha+\rho_{c} / \rho_{r}\right)$,
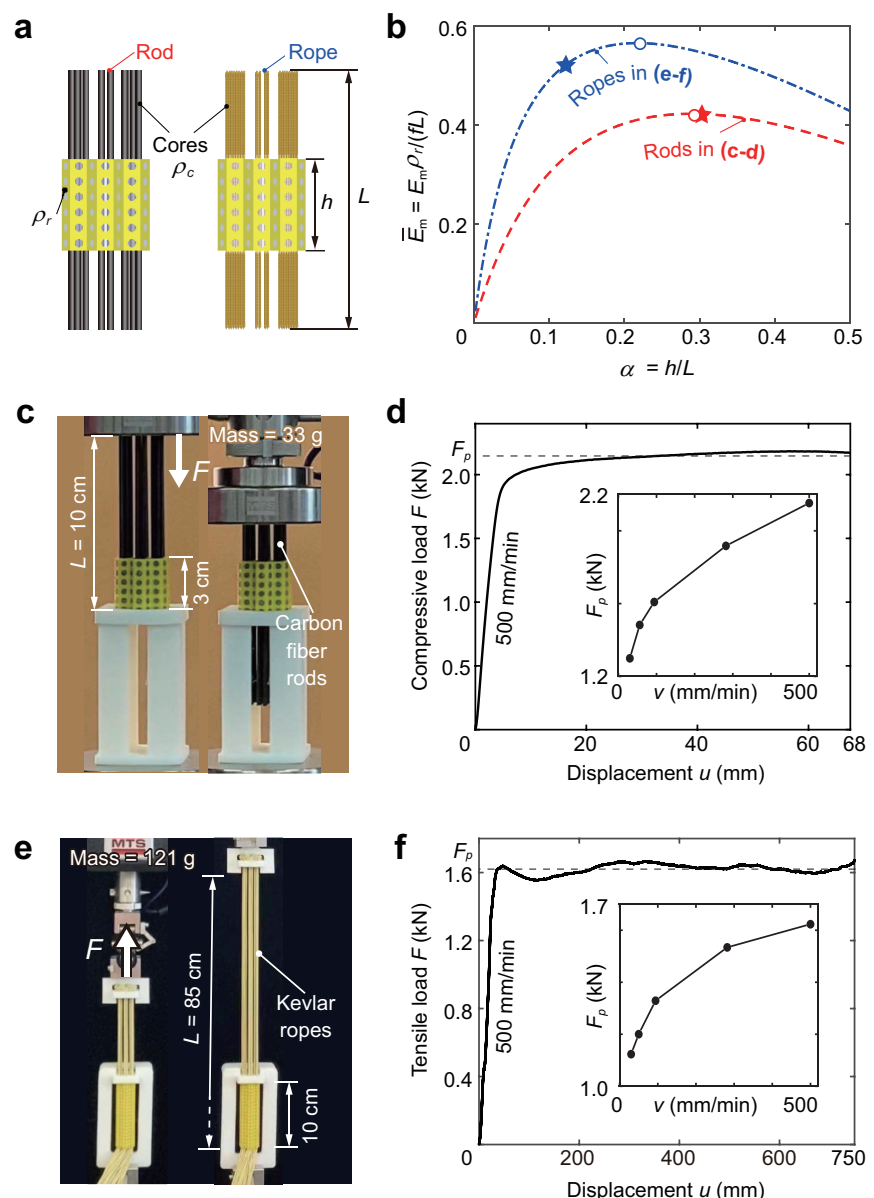

Fig. 4: Improving absorbed energy per unit mass. a Parameters of the metamaterials for calculating absorbed energy per unit mass. b $\bar{E}_{\mathrm{m}}-\alpha$ curves for metamaterials with different cores, where $E_{\mathrm{m}}$ is absorbed energy per unit mass. The hollow circles represent the maximal values of $\bar{E}_{\mathrm{m}}$, and the solid stars correspond to the parameters in the metamaterials shown in (c) and (e). c-d Metamaterials with carbon fiber rods as the cores and the corresponding loaddisplacement curve. e-f Metamaterials with Kevlar ropes as the cores and the corresponding load-displacement curve. The insets in (d) and (f) illustrate the dependence of the force plateau $F_{p}$ on the loading rate $v$.

which only depends on two dimensionless quantities $\alpha$ and $\rho_{c} / \rho_{r}$. While $\bar{E}_{\mathrm{m}}$ monotonically decreases with $\rho_{c} / \rho_{r}$, it non-monotonically increases and then decreases as $\alpha$ increases, reaching the maximal value at an optimal $\alpha$ (Fig. 4b).

Two samples were made to demonstrate the practical performance of the design. The first sample (Fig. 4c) used $2 \mathrm{~mm}$-diameter carbon fiber rods as cores. The length of the rods is $10 \mathrm{~cm}$ and the height of the elastomer is $3 \mathrm{~cm}$, so that the length ratio is $\alpha=0.3$. The corresponding dimensionless energy-absorbing quantity $\bar{E}_{\mathrm{m}}$ approaches the maximal value (see the red dashed line in Fig. 4b). This metamaterial only weights $33 \mathrm{~g}$, but can dissipate energy as large as $80 \sim 140 \mathrm{~J}$, depending on the loading rate (Fig. 4d). In other words, the energy-absorbing capacity 


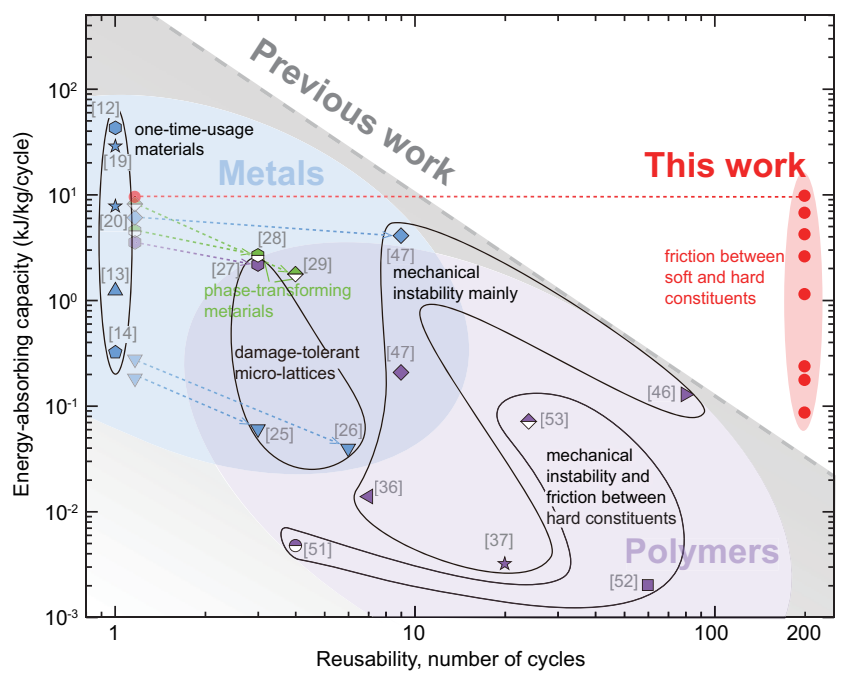

Fig. 5: Energy-absorbing capacity versus reusability of previous and current energy-absorbing materials. The cycle numbers are the maximum tested loops, and the dotted lines with arrows represent variation of energy-absorbing capacity with recycle number. Here, all points are calculated by experimental data from references ${ }^{12-14,19,20,25-29,36,37,46,47,51-53}$

reaches $2.6 \sim 4.2 \mathrm{~kJ} / \mathrm{kg}$, comparable to the non-reusable ones.

The second sample adopted Kevlar ropes as cores (Fig. 4e). In detail, we used a needle to thread two strands of $1.2 \mathrm{~mm}$-diameter Kevlar ropes into a $1.8 \mathrm{~mm}$-diameter elastomer hole. Since the ropes are more compliant, the prestress in this sample is lower than the first one. We enlarged $L$ to $850 \mathrm{~cm}$ (limited by the working stroke of our MTS machine) and $h$ to $10 \mathrm{~cm}$ to improve $E_{\mathrm{m}}$. The obtained length ratio is $\alpha=0.12$, and the corresponding $\bar{E}_{\mathrm{m}}$ is about $90 \%$ of the maximal value (see the blue dashed-dotted line in Fig. 4b). The metamaterial weights $121 \mathrm{~g}$, but can dissipate energy as large as $820 \sim 1190 \mathrm{~J}$, depending on the loading rate (Fig. 4e). In other words, the energy-absorbing capacity is $7 \sim 10 \mathrm{~kJ} / \mathrm{kg}$, which is even better than the first sample.

\section{Discussion}

capacity per cycle is multiplied by the number of repeated cycles to calculate the total energy-absorbing capacity in the entire life of a material, then a conservative estimate of our design is $10 \times 2000=2000 \mathrm{~kJ} / \mathrm{kg}$ per life, since the metamaterials are still intact and reusable after the tested cycles; and this value is at least 40 times as many as the others (Supplementary Fig. 6).

We attribute the extraordinary performance of the proposed metamaterials into two strategies of our design. Firstly, we utilized friction between soft elastomer and hard constituents rather than between hard particles, which is the strategy of the previous frictional metamaterials. Involving elastomer helps to achieve tightly contacted frictional interfaces that have a large amount of real contact area. Secondly, the interwoven structure strongly anchors the soft elastomer on the stiff supporting frame, which empowers the reinforced elastomer with robust load-bearing capability and applies larger prestress at the frictional interface. These interfaces notably improve the capacity of energy-absorbing metamaterials based on friction as shown in Fig. 5. In addition, the rate-dependent frictional behavior of elastomer interfaces enables the reported metamaterials passive adaptability to fit impacts with different velocities. We believe our design strategy opens a new technical path to obtain high-performance reusable energy-absorbing metamaterials.

\section{Methods}

Fabrication of the reinforced elastomer. The reinforced elastomer samples were manufactured by combing fused deposition modeling (FDM) 3D printing technology (Hori Z300 3D Printer) and mold-casting process. Materials adopted were commercial 3D printing PLA filaments (eSUN poly lactic acid), bright-finished 304 stainless steel rods of different diameters and a commercially available silicone elastomer (Dongguan ShinBon New Material Co., Ltd., China). The elastomer consists of two liquid constituent parts. They were mixed in a ratio of $1: 1$, then poured into 3D printed molds assembled with PLA frame and $1.8 \mathrm{~mm}$-diameter steel rods, and finally cured for 6 hours at room temperature. These steel rods were then removed after curing, and the corresponding holes left in the elastomer were subsequently inserted with thicker rods/ropes.

Measurements of the stress-strain curve of the silicone elastomer. To quantify the mechanical properties of the elastomer, uniaxial tension and compression tests were conducted using the single-axis MTS testing machine (E44.104). Due to the large deformation and low stiffness of the elastomer, video extensometry, instead of conventional clip-on extensometry, was used to measure the tensile strain. Specifically, a ruler was placed vertically on one side of the tension specimen, and the coordinates of the markers on the specimen were video recorded (see 
Supplementary Fig. 5).

Experimental tests of the proposed metamaterials. To characterize the energy absorption capacities of the metamaterials, uniaxial compression or uniaxial tension tests were performed. The force-displacement curves of the samples were measured under displacement control by a MTS testing machine. The loading rates were from $1 \mathrm{~mm} / \mathrm{min}$ to $500 \mathrm{~mm} / \mathrm{min}$, which is the maximal loading speed of the machine. In the experiments, assisting frames made of PLA were fabricated by 3D printing.

FEM simulations. In the present studies, several FEM models have been built in commercial software Abaqus/Standard 2014 to estimate the stress distribution in the silicone elastomer under loading. The elastomer was considered as incompressible, and its hyperelastic behavior was formulated by the third order Ogden material model with strain energy density

$$
W\left(\lambda_{1}, \lambda_{2}, \lambda_{3}\right)=\sum_{i=1}^{3} \frac{2 \mu_{i}}{\alpha_{i}^{2}}\left(\lambda_{1}^{\alpha_{i}}+\lambda_{2}^{\alpha_{i}}+\lambda_{3}^{\alpha_{i}}-3\right)
$$

where $\lambda_{1}, \lambda_{2}$, and $\lambda_{3}$ are principle stretches, and material parameters were adopted as $\mu_{1}=0.322 \mathrm{MPa}, \alpha_{1}=3.248$, $\mu_{2}=7.949 \times 10^{-5} \mathrm{MPa}, \alpha_{2}=13.706, \mu_{3}=6.667 \times$ $10^{-2} \mathrm{MPa}, \alpha_{3}=-3.490$. These parameters were determined by fitting against experimental data of uniaxial tensile and compression tests (for details see Supplementary Fig. 5).

In addition, Young's Modulus $E=3 \mathrm{GPa}$, Poisson's ratio $\nu=0.33$, and $E=206 \mathrm{GPa}, \nu=0.3$ were taken for the PLA frame and steel rod, respectively. Eight-node linear elements with hybrid formulation and reduced integration $(\mathrm{C} 3 \mathrm{D} 8 \mathrm{RH})$ were adopted for the silicone elastomer due to its incompressibility, and eight-node linear elements with reduced integration (C3D8R) were adopted for the PLA and steel rod. All geometric parameters of the model were consistent with those of the experimental samples.

\section{Acknowledgements}

This research at Tsinghua University was supported by National Natural Science Foundation of China (Grant No. 11872221, 12025203 and 11921002), and at University of California, Los Angeles was supported by the startup fund from Henry Samueli School of Engineering and Applied Science and Hellman fellowship. All the authors thank Meng $\mathrm{Li}$ at Tsinghua University for assisting the experiments.

\section{Author contributions}

Z.H.Z and J.Y.L. designed the research. Z.H.Z, L.H.J. and Q.Y.L. supervised the research. J.Y.L. made all of the metamaterial samples, and conducted related experiments and numerical simulations. J.Y.L and Z.C. measured frictional coefficient between elastomer and steel. All authors discussed the results and revised the manuscript at all stage.

\section{References}

1. Lu, G. \& Yu, T. Energy Absorption of Structures and Materials (Woodhead Publishing Ltd., Cambridge, 2003).

2. Connors, M. et al. Bioinspired design of flexible armor based on chiton scales. Nat. Commun. 10, 5413 (2019).

3. Davoodi, M. M., Sapuan, S. M. \& Yunus, R. Conceptual design of a polymer composite automotive bumper energy absorber. Mater. Des. 29, 1447-1452 (2008).

4. Caserta, G. D., Iannucci, L. \& Galvanetto, U. Shock absorption performance of a motorbike helmet with honeycomb reinforced liner. Compos. Struct. 93, 2748-2759 (2011).

5. Xu, F., Zhang, X. \& Zhang, H. A review on functionally graded structures and materials for energy absorption. Eng. Struct. 171, 309-325 (2018).

6. Bauer, J. et al. Nanolattices: an emerging class of mechanical metamaterials. Adv. Mater. 29, 1701850 (2017).

7. Li, S., Fang, H., Sadeghi, S., Bhovad, P. \& Wang, K. W. Architected origami materials: how folding creates sophisticated mechanical properties. Adv. Mater. 31, 1805282 (2019).

8. Alghamdi, A. A. A. Collapsible impact energy absorbers: an overview. Thin-Walled Struct. 39, 189213 (2001).

9. Ma, J. \& You, Z. Energy absorption of thin-Walled square tubes With a prefolded origami pattern - part I: geometry and numerical simulation. J. Appl. Mech.Trans. ASME 81, 011003 (2014).

10. Jiang, B. et al. Ultralight metal foams. Sci. Rep. $\mathbf{5}$, 13825 (2015).

11. Gibson, L. J. Mechanical behavior of metallic foams. Annu. Rev. Mater. Sci. 30, 191-227 (2000).

12. Xie, B., Fan, Y., Mu, T. \& Deng, B. Fabrication and energy absorption properties of titanium foam with $\mathrm{CaCl} 2$ as a space holder. Mater. Sci. Eng. AStruct. Mater. Prop. Microstruct. Process. 708, 419 423 (2017).

13. Fan, S. et al. Compressive properties and energy absorption characteristics of open-cell nickel foams. Trans. Nonferrous Met. Soc. China 27, 117-124 (2017).

14. Alvandi-Tabrizi, Y. \& Rabiei, A. Use of composite metal foam for improving absorption of collision forces. Procedia Mater. Sci. 4, 377-382 (2014). 
15. Meza, L. R., Das, S. \& Greer, J. R. Strong, lightweight, and recoverable three-dimensional ceramic nanolattices. Science 345, 1322-1326 (2014).

16. Bouville, F. \& Studart, A. R. Geologically-inspired strong bulk ceramics made with water at room temperature. Nat. Commun. 8, 14655 (2017).

17. Mao, A., Zhao, N., Liang, Y. \& Bai, H. Mechanically efficient cellular materials inspired by cuttlebone. $A d v$. Mater., 2007348 (2021).

18. He, M. et al. Development of a novel energy-absorbing bolt with extraordinarily large elongation and constant resistance. Int. J. Rock Mech. Min. Sci. 67, 29-42 (2014).

19. Stanev, L., Drenchev, B., Yotov, A. \& Lazarova, R. Compressive properties and energy absorption behaviour of AlSi10Mg open-cell foam. J. Mater. Sci. Technol. 22, 44-53 (2014).

20. Aldoshan, A. \& Khanna, S. Effect of relative density on the dynamic compressive behavior of carbon nanotube reinforced aluminum foam. Mater. Sci. Eng. A-Struct. Mater. Prop. Microstruct. Process. 689, 1724 (2017).

21. Banhart, J. Manufacture, characterisation and application of cellular metals and metal foams. Prog. Mater. Sci. 46, 559-632 (2001).

22. Ranjith, P. G. et al. Opportunities and challenges in deep mining: a brief review. Engineering 3, 546-551 (2017).

23. Mirkhalaf, M., Dastjerdi, A. K. \& Barthelat, F. Overcoming the brittleness of glass through bioinspiration and micro-architecture. Nat. Commun. 5, 3166 (2014).

24. Lee, S. W., Jafary-Zadeh, M., Chen, D. Z., Zhang, Y. W. \& Greer, J. R. Size effect suppresses brittle failure in hollow Cu60Zr40 metallic glass nanolattices deformed at cryogenic temperatures. Nano Lett. 15, 5673-5681 (2015).

25. Zheng, X. et al. Multiscale metallic metamaterials. Nat. Mater. 15, 1100-1106 (2016).

26. Schaedler, T. A. et al. Ultralight metallic microlattices. Science 334, 962-965 (2011).

27. Ling, C., Cernicchi, A., Gilchrist, M. D. \& Cardiff, P. Mechanical behaviour of additively-manufactured polymeric octet-truss lattice structures under quasistatic and dynamic compressive loading. Mater. Des. 162, 106-118 (2019).

28. Cooper, C. B. et al. Toughening stretchable fibers via serial fracturing of a metallic core. Sci. Adv. 5, eaat4600 (2019).
29. Cheng, Y., Li, J., Qian, X. \& Rudykh, S. 3D printed recoverable honeycomb composites reinforced by continuous carbon fibers. Compos. Struct. 268, 113974 (2021).

30. Deng, F., Nguyen, Q. K. \& Zhang, P. Multifunctional liquid metal lattice materials through hybrid design and manufacturing. Addit. Manuf. 33, 101117 (2020).

31. Coulais, C., Teomy, E., de Reus, K., Shokef, Y. \& van Hecke, M. Combinatorial design of textured mechanical metamaterials. Nature 535, 529-532 (2016).

32. Chen, Y., Li, X., Hu, G., Haberman, M. R. \& Huang, G. An active mechanical Willis meta-layer with asymmetric polarizabilities. Nat. Commun. 11, 3681 (2020).

33. Reeve, S. T., Belessiotis-Richards, A. \& Strachan, A. Harnessing mechanical instabilities at the nanoscale to achieve ultra-low stiffness metals. Nat. Commun. 8, 1137 (2017).

34. Zhao, H. et al. Buckling and twisting of advanced materials into morphable 3D mesostructures. Proc. Natl. Acad. Sci. U. S. A. 116, 13239-13248 (2019).

35. Rafsanjani, A., Akbarzadeh, A. \& Pasini, D. Snapping mechanical metamaterials under tension. Adv. Mater. 27, 5931-5935 (2015).

36. Haghpanah, B., Salari-Sharif, L., Pourrajab, P., Hopkins, J. \& Valdevit, L. Multistable shapereconfigurable architected materials. Adv. Mater. 28, 7915-7920 (2016).

37. Shan, S. et al. Multistable architected materials for trapping elastic strain energy. Adv. Mater. 27, 42964301 (2015).

38. Tan, X. et al. Reusable metamaterial via inelastic instability for energy absorption. Int. J. Mech. Sci. 155, 509-517 (2019).

39. Chen, S. et al. A novel composite negative stiffness structure for recoverable trapping energy. Compos. Part A Appl. Sci. Manuf. 129, 105697 (2020).

40. Alturki, M. \& Burgueno, R. Response characterization of multistable shallow domes with cosine-curved profile. Thin-Walled Struct. 140, 74-84 (2019).

41. Hewage, T. A., Alderson, K. L., Alderson, A. \& Scarpa, F. Double-negative mechanical metamaterials displaying simultaneous negative stiffness and negative Poisson's ratio properties. Adv. Mater. 28, 10323-10332 (2016).

42. Chen, T. et al. Harnessing magnets to design tunable architected bistable material. Adv. Eng. Mater. 21, 1801255 (2019).

43. Tan, X. et al. Design, fabrication, and characterization of multistable mechanical metamaterials for trapping energy. Extreme Mech. Lett. 28, 8-21 (2019). 
44. Pan, F. et al. 3D pixel mechanical metamaterials. Adv. Mater. 31, 1900548 (2019).

45. Chen, Y. \& Jin, L. Reusable energy-absorbing architected materials harnessing snapping-back buckling of wide hyperelastic columns. Adv. Funct. Mater. 31, 2102113 (2021).

46. Frenzel, T., Findeisen, C., Kadic, M., Gumbsch, P \& Wegener, M. Tailored buckling microlattices as reusable light-weight shock absorbers. Adv. Mater. 28, 5865-5870 (2016).

47. Zhu, S. et al. Bio-inspired multistable metamaterials with reusable large deformation and ultra-high mechanical performance. Extreme Mech. Lett. 32, 100548 (2019).

48. Zhu, S. et al. A novel bi-material negative stiffness metamaterial in sleeve-type via combining rigidity with softness. Compos. Struct. 262, 113381 (2021).

49. Hua, J., Lei, H., Gao, C., Guo, X. \& Fang, D. Parameters analysis and optimization of a typical multistable mechanical metamaterial. Extreme Mech. Lett. 35, 100640 (2020).

50. Duoss, E. B. et al. Three-dimensional printing of elastomeric, cellular architectures with negative stiffness. Adv. Funct. Mater. 24, 4905-4913 (2014).

51. Fu, K., Zhao, Z. \& Jin, L. Programmable granular metamaterials for reusable energy absorption. Adv. Funct. Mater. 29, 1901258 (2019).

52. Garland, A. P. et al. Coulombic friction in metamaterials to dissipate mechanical energy. Extreme Mech. Lett. 40, 100847 (2020).

53. Wang, Y. et al. Architected lattices with adaptive energy absorption. Extreme Mech. Lett. 33, 100557 (2019).

54. Riley, G. Tendinopathy-from basic science to treatment. Nat. Clin. Pract. Rheumatol. 4, 82-89 (2008).

55. Schuenke, M., Schulte, E. \& Schumacher, U. Thieme Atlas of Anatomy: General Anatomy and Musculoskeletal System (Thieme Medical Publishers, Incorporated, New York, 2010).

56. Duan, S. et al. Enhanced out-of-plane compressive strength and energy absorption of 3D printed square and hexagonal honeycombs with variable-thickness cell edges. Extreme Mech. Lett. 18, 9-18 (2018).

57. Kapfer, S. C., Hyde, S. T., Mecke, K., Arns, C. H. \& Schroder-Turk, G. E. Minimal surface scaffold designs for tissue engineering. Biomaterials 32, 6875-6882 (2011).

58. Persson, B. N. J. \& Volokitin, A. I. Theory of rubber friction: Nonstationary sliding. en. Phys. Rev. B 65, 134106 (2002).
59. Grosch, K. A. Relation between the friction and viscoelastic properties of rubber. Nature 197, 858-859 (1963).

60. Bouchbinder, E., Brener, E. A., Barel, I. \& Urbakh, M. Slow cracklike dynamics at the onset of frictional sliding. en. Phys. Rev. Lett. 107, 235501 (2011).

61. Thogersen, K., Gilbert, A., Schuler, T. V. \& MaltheSorenssen, A. Rate-and-state friction explains glacier surge propagation. Nat. Commun. 10, 2823 (2019).

62. Popov, V. L., Voll, L., Kusche, S., Li, Q. \& Rozhkova, S. V. Generalized master curve procedure for elastomer friction taking into account dependencies on velocity, temperature and normal force. Tribol. Int. 120, 376-380 (2018).

63. Bergstrom, J. S. Mechanics of Solid Polymers: Theory and Computational Modeling (William Andrew, San Diego, 2015).

64. Grosch, K. A. \& Bowden, F. P. The relation between the friction and visco-elastic properties of rubber. Proc. R. Soc. London, Ser. A 274, 21-39 (1963). 


\section{Supplementary Files}

This is a list of supplementary files associated with this preprint. Click to download.

- sourcedata.xlsx

- SupplementaryInformation.docx

- SupplementaryMovie1.mp4

- SupplementaryMovie2.mp4 